\title{
From a Low Cost Spectrograph to a High Resolution Spectrograph
}

\author{
T. T. Grove \\ Department of Physics, Indiana University Purdue University Fort Wayne, 2101 E. Coliseum Blvd, Fort Wayne, IN \\ 46805

\begin{abstract}
We have been using low-cost spectrographs called shoebox spectrographs for a few years. In the process of our study, we decided to make a spectrograph using the same basic optical design but with quality optical parts. This new spectrograph was found to be easily aligned, very accurate ( 0.2 Angstrom accuracy), and enables intermediate and advanced students to study molecular spectral lines. We will present theory of operation as well as accurate photographs of molecular spectra.
\end{abstract}

Keywords: Spectra, Advanced Lab, Spectrograph

PACS: 07.57.-c, 07.60.Rd

\section{INTRODUCTION}

Several years ago, we developed a low cost spectrograph (shoebox spectrograph) for the purpose of discussing spectral features of light with undergraduate students. Each spectrograph unit costs $\sim \$ 30$ with most of that cost being the webcam (Logitech model c310). Figure 1 shows a photograph of this spectrograph (sans webcam) and Fig. 2 shows the relevant optical components. The shoebox spectrograph is accurate to within $\sim 2 \mathrm{~nm}$ of identifying wavelengths for atomic spectra which we feel is impressive given its component parts.

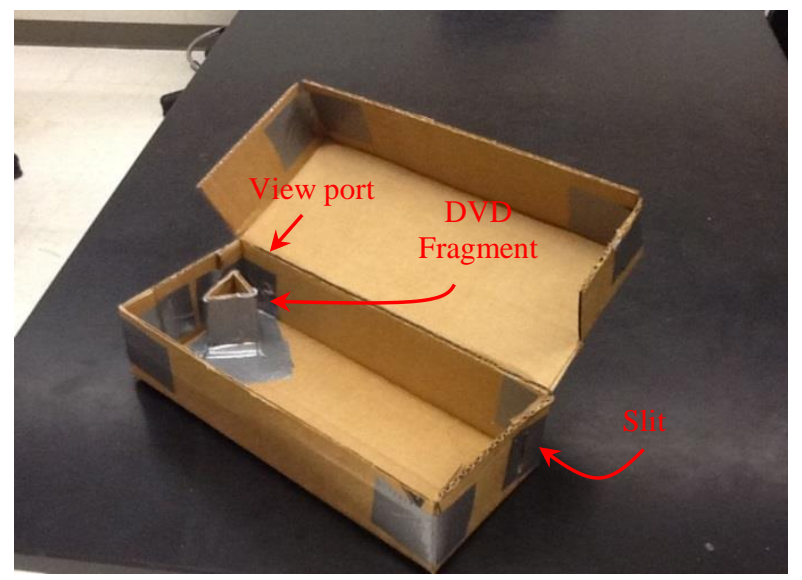

FIGURE 1. A photograph of the shoebox spectrograph. It is approximately $30 \mathrm{~cm}$ by $12 \mathrm{~cm}$ by $7 \mathrm{~cm}$ and is constructed using scrap corrugated cardboard, duct tape, school glue, and a DVD fragment for a grating. The slit and viewport are literally cut into the cardboard using cutting tools (utility knife and robber stopper hole cutter)

Pre-cursors to the shoebox spectrograph were made without a full optical explanation. That is, we made similar boxes and to our surprise, they worked far better than we could have hoped. For example, most spectrographs use carefully constructed slits while ours used a crude cut hole (which seems altogether too wide to provide narrow spectral features). In searching for how the shoebox spectrograph functions, we first developed an optical explanation and then from this explanation we wanted to see what one could really do with this optical design given better components (which is the basis of this report).

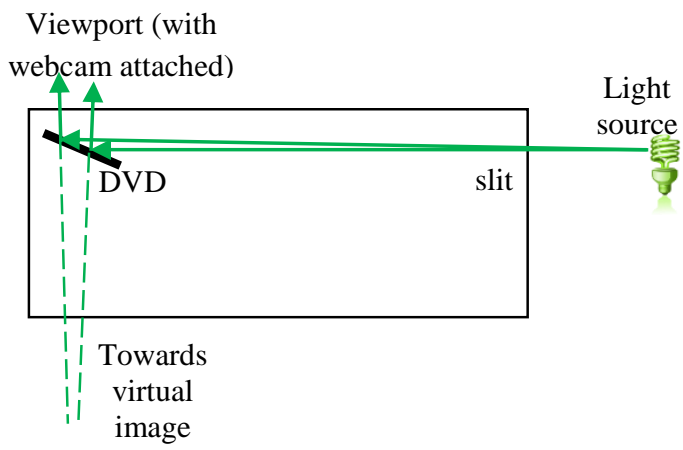

FIGURE 2. Sketch of the internal components of the shoebox spectrograph. A webcam is attached to the side of the box in order to take spectral accurate photographs.

\section{OPTICAL EXPLANATION}

The physical mechanism behind the shoebox spectrograph is very reminiscent of the introductory physics explanation for how a plane mirror forms a virtual image. The big difference is that we replace the plane mirror with a diffraction grating which makes the math a bit more complicated. Regardless, light passing 
through a slit is diffracted off a grating as shown in Fig. 3 with only the $1^{\text {st }}$ order diffraction shown for simplicity.

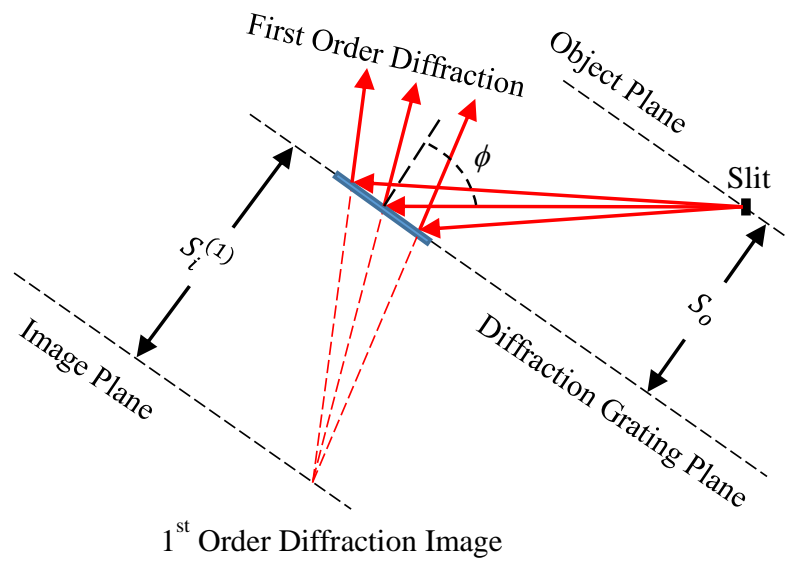

FIGURE 3. Ray schematic for the shoebox spectrograph. Because each ray passing through the slit has a slightly different incidence angle with respect to the grating, each ray diffracts at slightly different angles. The diverging rays emanating from the slit form a virtual image behind the grating.

A set of rays emanating from the slit diffract from the grating and diverge (due to each ray having a slightly different incidence angle). The diffraction equation ${ }^{1}$ [given by

$$
d\left(\sin \theta_{i}+\sin \theta_{m}\right)=m \lambda
$$

where $d$ is the distance between repeating features on the grating, $\theta_{i}$ is the incidence angle, $m$ is the order of diffraction, $\theta_{m}$ is the diffraction angle, and $\lambda$ is the wavelength] gives us the angle at which each ray diffracts. With the diffraction equation and use of the paraxial approximation, we can determine the distance at which the image plane forms behind the grating plane. It is given by the equation

$$
S_{i}^{(m)}=\frac{S_{0}\left[d^{2}-(m \lambda-d \sin \phi)\right]^{\frac{3}{2}}}{d^{3} \cos ^{3} \phi}
$$

where $\phi$ is the center ray's angle of incidence with the grating. The most important part of equation (2) is the cosine term in the denominator. When the incidence angle approaches grazing incidence, the image distance (distance from grating plane to image plane) approaches infinity. For the shoebox spectrograph our $\phi$ value is approximately $77^{\circ}$. Since the grating spacing for a DVD is $d=740 \mathrm{~nm},{ }^{2}$ we find that the image forms more than 3 meters behind the grating. With further investigation we found that if we incorporate the slit width into our model, our large width slit $(\sim 2 \mathrm{~mm})$ covers little of the spectrum; the width of the image is very small compared to the distance from the image plane and grating plane. Thus, the image of the slit (still a few millimeters wide) seen as being a few meters away helps explain how our crude slit is still yields narrow line widths.

\section{OPTICAL SET UP FOR THE HIGH RESOUTION SPECTROGRAPH}

Given our understanding of the shoebox spectrograph, we now constructed a more powerful spectrograph based upon its design. Figure 4 shows the basic set up. A pair of lens are used to maximize the light passing through the slit. Then an achromat lens is used to collimate the light after the slit. Its purpose is to effectively make $S_{0}$ approach infinity for equation (2). Instead of using a DVD, this time we used an off the shelf diffraction grating (1200 grooves/mm). Following the grating, we used a long focal length achromat lens. This enables us to magnify the real image that forms on the camera. Because the image of the spectral lines far exceeds the size of the photosensitive detector inside the camera, the grating is on a precision rotation stage. This allows us to select which part of the spectra is photographed.

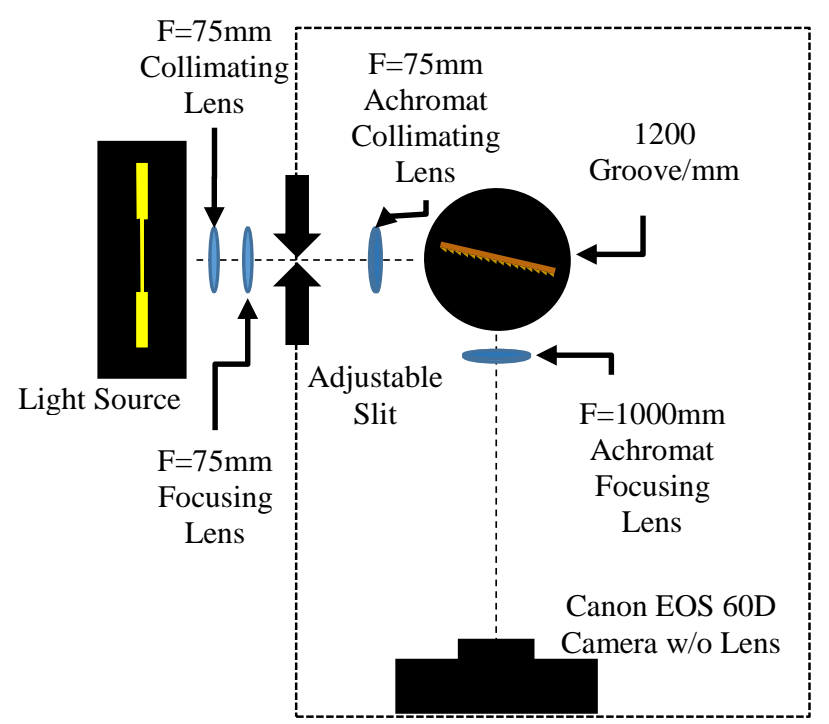

FIGURE 4. Light from a source is first collimated and then focused through a slit. After the slit the light is again collimated. The light is then diffracted off the grating and focused into a camera (with lens removed) with a long focal length lens.

The alignment of the spectrograph is actually very easy and straightforward. We start by using a sodium light source due to its brightness. The grating is rotated so that the sodium D-lines (brightest lines in the sodium spectrum) diffract off the grating making a right angle with the incident direction (as seen by eye). The $1000 \mathrm{~mm}$ lens is then added and one can follow the D- 
lines to the camera with the use of a business card. Finally, the camera is switched to video mode and the last distance adjustments can be made while watching the camera's LCD screen (to ensure the best possible focus).

This spectrograph bears many similarities to grazing incidence spectrograph used to record ultra violet light. ${ }^{3}$ However, there are differences. The grazing incidence spectrographs in common use do not have an achromat collimating lens and the gratings used are concave which eliminates the need for focusing optics. These changes allow this spectrograph to work well within the visible region and provide good results that undergraduates can assemble in lab.

\section{RESULTS}

By taking a photograph, rotating the grating by half a degree, taking another photograph, and then continuing the process, we can record the entire visible spectrum. Features on one photograph align with features from preceding and subsequent photos. In this way a grand compilation of the entire spectrum is formed.

We have also found that replacing or moving the light source has minimal effect on the data. Thus, by using a well-known spectrum like neon we can calibrate our results. Figure 5, shown below, helps illustrate the repeatability of our spectrograph.

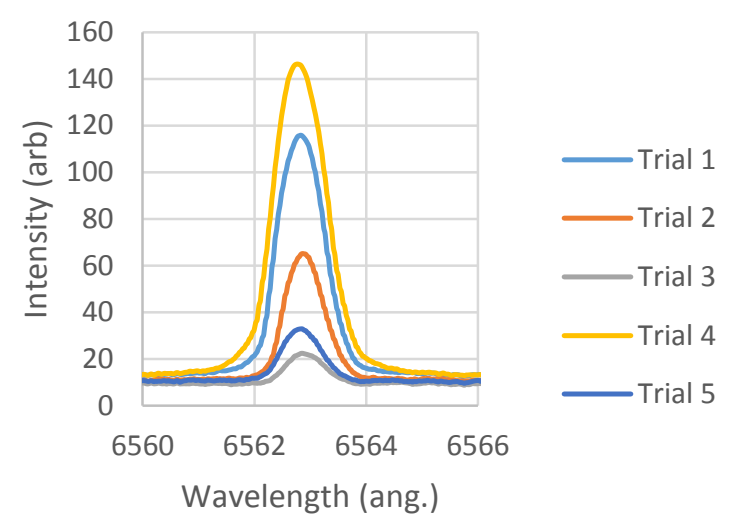

FIGURE 5. Multiple traces of Hydrogen's red Balmer line. Between each trial, the light source was replaced and moved. The traces shown above help illustrate the reliability of each photograph. The horizontal axis is based upon a separate set of photographs of the Neon spectrum. The known wavelength of the red Balmer line was not used for calibration.

Each trace represents a different picture of the hydrogen red Balmer line ( $\mathrm{H}-\alpha$ as it is known historically) where the hydrogen source was literally moved and then replaced by a different hydrogen source. All the traces are from photographs which have synchronized pixels, but if one looks deeply enough at the peak location of all the traces there small differences. The peak location of the traces ranged from a low of 6562.77 angstroms to a high of 6562.87 angstroms. The accepted value of the peak location is 6562.8518 angstroms (with two smaller nearby peaks at 6562.7110 angstroms and 6562.7248 angstroms). ${ }^{4}$

To help demonstrate this spectrograph's capabilities, Figure 6 shows a compilation of nitrogen spectra across the entire visible spectrum. We know the corresponding wavelengths of each of the lines by comparison back to an equivalent neon spectrum. A small portion of the entire spectrum is shown in Fig. 7. This intensity vs. wavelength graph is only a very small piece of the entire spectrum, but it shows all the spectral lines seen inside the magnifying glass.

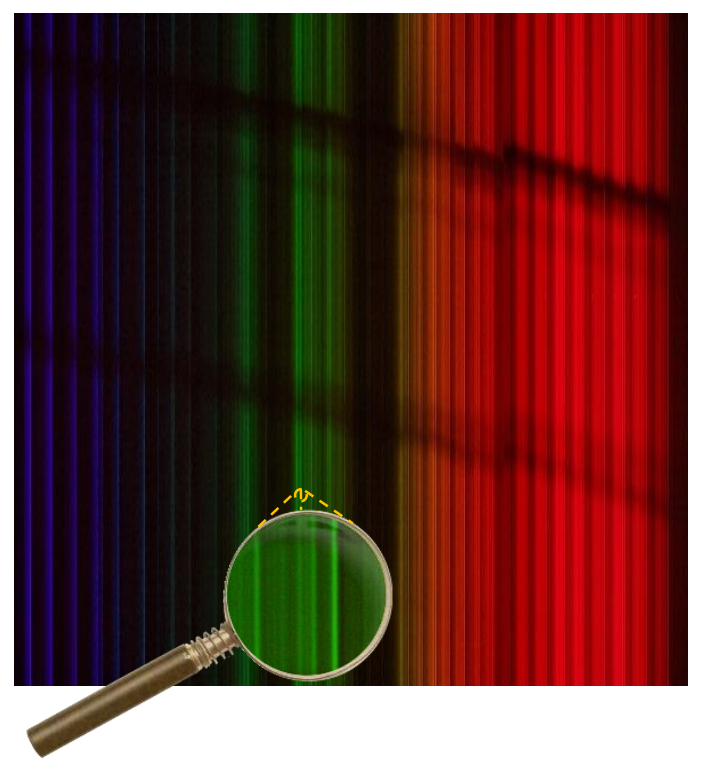

FIGURE 6. Compiled nitrogen spectrum. The broad bands as seen in the larger picture are actually composed of many spectral lines. The "magnifying glass" was added to show some of the finer lines. The small spectral lines seen inside the "magnifying glass" come from the small region illustrated on the larger picture.

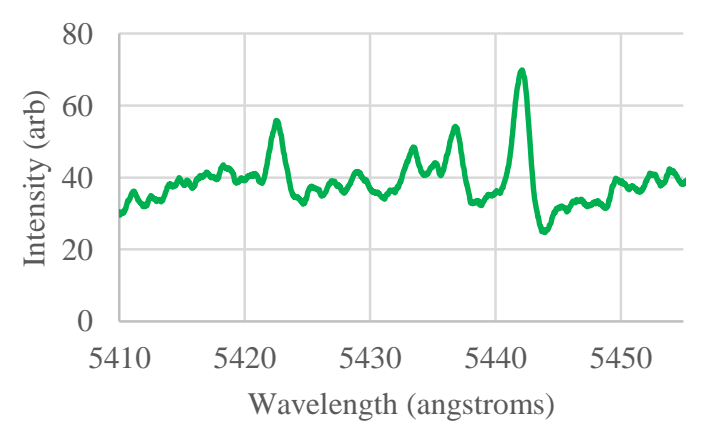


FIGURE 7. Intensity vs. wavelength for the part of the nitrogen spectra seen inside the magnifying glass in Fig. 5.

\section{CONCLUSION}

We have created a fairly powerful spectrograph that undergraduates can construct to observe molecular spectra (like the nitrogen spectra seen above). It is easy to align and use. Furthermore, it is likely that the overall cost can be reduced. The Canon EOS 60d camera is the

${ }^{1}$ For Example see, F. L. Pedrotti and L. S. Pedrotti, Introduction to Optics (Prentice-Hall International, Englewood, NJ, 1987), p. 418.

${ }^{2}$ Fumitaka Wakabayashi, J. Chem. Educ. 85, 849 - 853 (2008) most expensive part $(\sim 650)$, but it could be replaced by cheaper cameras (for example, cameras designed for telescopes).

${ }^{3}$ For example see, H. A. Kirkpatrick, J. Quart. Spectrosc. Radiat. Transfer, Vol. 2 pp. 715 - 724 (1962).

${ }^{4}$ See,

http://physics.nist.gov/PhysRefData/Handbook/Tables/hy drogentable $2 . \mathrm{htm}$ 